\title{
Female Reproductive System Precancerous Condition
}

National Cancer Institute

\section{Source}

National Cancer Institute. Female Reproductive System Precancerous Condition. NCI

Thesaurus. Code C27788.

A precancerous lesion that arises from the female reproductive system. Representative examples include atypical endometrial hyperplasia, endometrial intraepithelial neoplasia, and cervical intraepithelial neoplasia. 